\title{
Integrated bioinformatics analysis for the identification of potential key genes affecting the pathogenesis of clear cell renal cell carcinoma
}

\author{
HAO CUI ${ }^{1,2 *}$, LEI XU ${ }^{3 *}, \mathrm{ZHI} \mathrm{LI}^{1,2},{\mathrm{KE}-\mathrm{ZUO} \mathrm{HOU}^{1,2}, \mathrm{XIAO-FANG} \mathrm{CHE}}^{1,2}$, \\ BO-FANG LIU ${ }^{1,2}$, YUN-PENG LIU ${ }^{1,2}$ and XIU-JUAN QU ${ }^{1,2}$ \\ ${ }^{1}$ Department of Medical Oncology; ${ }^{2}$ Key Laboratory of Anticancer Drugs and Biotherapy of Liaoning Province; \\ ${ }^{3}$ Department of Breast Surgery, The First Hospital of China Medical University, Shenyang, Liaoning 110001, P.R. China
}

Received September 13, 2019; Accepted April 15, 2020

DOI: $10.3892 / \mathrm{ol} .2020 .11703$

\begin{abstract}
Clear cell renal cell carcinoma (CCRCC) is a typical type of RCC with the worst prognosis among the common epithelial neoplasms of the kidney. However, its molecular pathogenesis remains unknown. Therefore, the aim of the present study was to screen for effective and potential pathogenic biomarkers of CCRCC. The gene expression profile of the GSE16441, GSE36895, GSE40435, GSE46699, GSE66270 and GSE71963 datasets were downloaded from the Gene Expression Omnibus database. First, the limma package in $\mathrm{R}$ language was used to identify differentially expressed genes (DEGs) in each dataset. The robust and strong DEGs were explored using the robust rank aggregation method. A total of 980 markedly robust DEGs were identified (429 upregulated and 551 downregulated). According to Gene Ontology and Kyoto Encyclopedia of Genes and Genomes pathway enrichment analysis, these DEGs exhibited an obvious enrichment in
\end{abstract}

Correspondence to: Professor Xiu-Juan $\mathrm{Qu}$ or Professor Yun-Peng Liu, Department of Medical Oncology, The First Hospital of China Medical University, 155 North Nanjing Street, Shenyang, Liaoning 110001, P.R. China

E-mail: xiujuanqu@yahoo.com

E-mail: cmuliuyunpeng@hotmail.com

${ }^{*}$ Contributed equally

Abbreviations: RCC, renal cell carcinoma; CCRCC, clear cell renal cell carcinoma; GEP, gene expression profile; GEO, Gene Expression Omnibus; DEGs, differentially expressed genes; RRA, robust rank aggregation; KEGG, Kyoto Encyclopedia of Genes and Genomes; GO, Gene Ontology; PPI, protein-protein interaction; TCGA, The Cancer Genome Atlas; FC, fold-change; DAVID, Database for Annotation, Visualization and Integrated Discovery; $\mathrm{MF}$, molecular function; $\mathrm{BP}$, biological process; $\mathrm{CC}$, cellular component; MCC, Maximal Clique Centrality; MNC, Maximum Neighborhood Component

Key words: CCRCC, GEO, DEGs, RRA, biomarkers, bioinformatics various cancer-related biological pathways and functions. The Search Tool for the Retrieval of Interacting Genes/Proteins database was used for the construction of a protein-protein interaction (PPI) network, the Cytoscape MCODE plug-in for module analysis and the cytoHubba plug-in to identify hub genes from the aforementioned DEGs. A total of four key modules were identified in the PPI network. A total of six hub genes, including $\mathrm{C}-\mathrm{X}-\mathrm{C}$ motif chemokine ligand 12, bradykinin receptor $\mathrm{B} 2$, adenylate cyclase 7 , calcium sensing receptor (CASR), kininogen 1 and lysophosphatidic acid receptor 5, were identified. The DEG results of the hub genes were verified using The Cancer Genome Atlas database, and CASR was found to be significantly associated with the prognosis of patients with CCRCC. In conclusion, the present study provided new insight and potential biomarkers for the diagnosis and prognosis of CCRCC.

\section{Introduction}

Renal cell carcinoma (RCC) originally develops in the renal epithelium and accounts for $>90 \%$ of kidney cancer cases (1). In total, $>30 \%$ of patients with RCC are diagnosed with locally advanced and metastatic disease (2). Among RCC cases clear cell RCC (CCRCC) is a typical histologic type, accounting for $80-90 \%$ of cases (3). Although surgical resection can effectively resolve CCRCC, 20-40\% of patients still develop metastasis or recurrence following surgery (4). In addition, CCRCC has the worst prognosis among the common epithelial kidney tumors (5). Recently, significant progress has been made in the current understanding of CCRCC development; for example, the von Hippel-Lindau tumor suppressor gene is inactivated frequently by genetic alteration (6). However, the molecular mechanisms underlying CCRCC pathogenesis remain unknown. Therefore, the aim of the present study was to screen for effective and potential pathogenic biomarkers of CCRCC.

Recently, microarrays and RNA-sequencing on high-throughput platforms and public databases, such as Gene Expression Omnibus (GEO) and The Cancer Genome Atlas (TCGA), have emerged as novel and potential methods to examine significant genetic alternations in carcinogenesis. 
These methods have been applied to identify promising biomarkers for the diagnosis and prognosis of several types of human cancer, including CCRCC. Song et al (7) used a total of six GEO datasets with gene expression profiles (GEPs) of CCRCC and identified 129 up- and 123 downregulated genes, which were associated with signal transduction, metabolism and immune system pathways. Another previous study used the GSE53000 dataset to identify 533 up- and 642 downregulated differentially expressed genes (DEGs). A total of 10 genes were identified as hub genes of CCRCC, namely DNA topoisomerase II $\alpha$ (TOP2A), matrix metalloproteinase 9 (MMP-9), albumin, cyclin-dependent kinase 1, MYC, vascular endothelial growth factor A (VEGFA), calcium sensing receptor (CASR), protein tyrosine phosphatase receptor type C, prostaglandin-endoperoxide synthase 2 and endothelial growth factor receptor. A total of six CCRCC-related modules were identified in co-expression networks, which were associated with metabolic processes, immunoreaction, cell cycle regulation, ion transport and angiogenesis (8). Furthermore, based on weighted gene co-expression network analysis, Chen et al (9) identified 29 hub genes that exhibited a significant positive correlation with CCRCC progression at four stages of CCRCC. These genes could also serve as prognostic, recurrence or progression biomarkers of CCRCC.

However, the use of different experimental platforms, limited sample sizes and inappropriate analysis methods lead to outliers and inconsistent results. Therefore, unbiased and integrated approaches are necessary. The robust rank aggregation (RRA) method can identify genes exhibiting a better-than-expected performance according to the null hypothesis of uncorrelated inputs and robust regarding the result errors and noise. This method can reduce noise compared to signal while integrating data information of different platforms, which can make the research results more reliable (10). The RRA method integrates multiple gene expression datasets well, which improves high-throughput characterization of novel tumor genes with regard to their molecular mechanisms. This method was recently reported in studies focusing on several types of cancer, included gastric (11), bladder (12), non-small cell lung (13) and ovarian cancer (14). However, to the best of the authors' knowledge, no study to date has used this integration approach to investigate CCRCC.

In the present study, an integrated bioinformatics analysis was conducted to identify promising biomarkers for the diagnosis and prognosis of CCRCC. According to the GEPs of GEO datasets, 980 markedly robust DEGs (including 429 up- and 551 downregulated) were identified using the RRA method. Functional biological analysis suggested that these DEGs exhibited a marked enrichment in cancer-related biological pathways and functions. A protein-protein interaction (PPI) network was constructed using the Search Tool for the Retrieval of Interacting Genes/Proteins (STRING) database. The Cytoscape MCODE plug-in helped perform module analysis of the whole network. In total, six hub genes, C-X-C motif chemokine ligand 12 (CXCL12), bradykinin receptor B2 (BDKRB2), adenylate cyclase 7 (ADYC7) CASR, kininogen 1 (KNG1) and lysophosphatidic acid receptor 5 (LPAR5) were identified and verified in TCGA database. Moreover, it was identified that CASR exhibited marked association with CCRCC prognosis, as suggested by the survival analysis.
To the best of the authors' knowledge, the present study constituted the first time the RRA algorithm was used to investigate CCRC. Moreover, key genes were identified through bioinformatics methods, including analysis associated with clinical pathology and prognosis survival. The accuracy of the bioinformatics results of the present study was further confirmed by experimental verification methods. In conclusion, the present study provided new insight and promising potential biomarkers for CCRCC diagnosis and prognosis.

\section{Materials and methods}

CCRCC GEPs and data processing. The GEPs of CCRCC GSE16441 (15), GSE36895 (16), GSE40435 (17), GSE46699 (18), GSE66270 (19) and GSE71963 (20) were downloaded from the GEO database (https://www.ncbi. nlm.nih.gov/geo/). The screening criteria were as follows: i) CCRCC; ii) samples containing tumor and paired normal tissues; iii) array-based gene expression profiling as the study type; iv) Homo sapiens as the organism; and v) sample size $>20$. Supplementary information on the aforementioned six GEO datasets is listed in detail in Table SI. If multiple probes responded to any given gene, the median expression level of the gene was regarded as the final expression. Finally, quantile normalization was performed to standardize the gene expression levels (Fig. S1).

$D E G$ screening in GEO datasets. In order to investigate DEGs in every GEO dataset, the limma package v.3.42.2 (21) in R language was used to compare tumor and normal tissues with the cut-off set at $\log _{2} \mathrm{FCl}>1$ and modified $\mathrm{P}<0.05$, where $\mathrm{FC}$ indicates fold change. All gene list results, which were categorized according to $\log _{2}(\mathrm{FC})$ in every dataset, were preserved for later analysis.

RRA method. To find consistent and robust DEGs among the GEO datasets, the RRA method was used, which can identify genes with a better-than-expected performance on the basis of the null hypothesis of uncorrelated inputs and is robust to result errors and noise. RRA was carried out using the $\mathrm{R}$ package RobustRankAggreg v.1.1 (10). A $\mid \log _{2} \mathrm{FCl}>1$ and corrected $\mathrm{P}<0.05$ was considered to indicate a statistically significant difference for robust DEGs. A comprehensive list of up- and downregulated DEGs used for further analysis is presented in Table SII.

Gene ontology (GO) term and kyoto encyclopedia of genes and genomes (KEGG) pathway enrichment analysis. With the aim of examining the important biological function of the identified DEGs, the Database for Annotation, Visualization and Integrated Discovery (DAVID; version 6.8; https://david. ncifcrf.gov/) was used to perform enrichment analysis on GO terms (22). The GO terms included molecular function (MF), biological process (BP) and cellular component (CC) with a false discovery rate cut-off value $<0.05$. The KEGG Orthology Based Annotation System (KOBAS) online analysis database (version 3.0; http://kobas.cbi.pku.edu.cn/) (23) was used for KEGG pathway enrichment analysis of DEGs, with a corrected $\mathrm{P}<0.05$. 
PPI network building and module analysis. A PPI network was constructed using STRING (version 10.5; http://string-db. org/) (24). The interaction parameter was set as a maximum confidence of 0.90. Cytoscape software (version 3.6.1; http://www.cytoscape.org/) was used to visualize and analyze the PPI network. The Cytoscape MCODE plug-in v.1.6 (25), which can automatically identify the molecular complexes in a large PPI network, was used to screen for key modules from the whole network with parameters set to default (false degree cut-off, 2; K-Core, 2; haircut, true; node score cut-off, 0.2; fluf, false; maximum depth from seed, 100).

Identification of hub genes. The cytoHubba v.0.1 (26) plug-in was used to select potential hub genes from the identified DEGs. The Maximal Clique Centrality (MCC) as well as Maximum Neighborhood Component (MNC) analysis was used to identify the hub genes, with the shared hub genes screened using a Venn diagram. The two aforementioned plug-ins were derived from Cytoscape MCODE plug-in v.1.6.

TCGA-based validation and prognostic analysis. The fragments per kilobase per million value was used to determine up- or downregulation of GEPs of CCRCC from TCGA database (https://cancergenome.nih.gov/) helped validate the DEG results obtained from 72 pairs of tumor and normal tissues. The expression heat map and violin plots were used to represent tumors and normal samples exhibiting different expression patterns. The Gene Expression Profiling Interactive Analysis (GEPIA) online analysis tool (http://gepia.cancer-pku.cn/) (27) was used to analyze the prognosis and stage of hub genes. Pearson's correlation coefficient $(\mathrm{R})$ was calculated to evaluate the association among these hub genes. $\mathrm{P}<0.05$ was considered to indicate a statistically significant difference.

Tissue sample collection. All tissue samples were obtained from The Shengjing Hospital of China Medical University, including 20 pairs of CCRCC and matched normal tissue. Patients who underwent curative surgery from July 2003 to September 2007 without receiving any pre-operative chemotherapy, radiotherapy or targeted therapy prior to resection, were included in the present study. Patients with metastases were excluded. CCRCC diagnosis was confirmed by a pathologist using histochemical examination of tumor biopsies. Pathological grading of the tumor samples was based on the 7th TNM staging (28). Tissue samples were frozen in liquid nitrogen immediately after surgical resection and stored at $-80^{\circ} \mathrm{C}$ before further use. Informed consent forms were signed by all patients prior to sample collection. The present study was approved by The Research Ethics Committee of China Medical University (approval no. 2015PS44K).

RNA isolation and reverse transcription-quantitative PCR $(R T-q P C R)$. Total RNA was extracted from CCRCC and normal tissue using TRIzol ${ }^{\circledR}$ (Invitrogen; Thermo Fisher Scientific, Inc.) according to the manufacturer's instructions. Total RNA was solubilized in RNase-free water, and the concentration and purity of the samples were determined using a NanoDrop ${ }^{\mathrm{TM}} 2000$ spectrophotometer (Thermo Fisher Scientific, Inc.). RT was performed using a reverse transcription cDNA kit (Thermo First Strand cDNA Synthesis kit
(Thermo Fisher Scientific, Inc.) with the following conditions: $42^{\circ} \mathrm{C}$ for $60 \mathrm{~min}, 70^{\circ} \mathrm{C}$ for $5 \mathrm{~min}$, then $4^{\circ} \mathrm{C}$ preservation. SYBR ${ }^{\circledR}$ Green Fast qPCR Mix (High ROX) (Servicebio) was used for RT-qPCR with the StepOne Plus PCR system (Applied Biosystems; Thermo Fisher Scientific, Inc.). Thermocycling conditions were as follows: Pre-denaturation at $95^{\circ} \mathrm{C}$ for $10 \mathrm{~min}$, followed by 40 cycles of $95^{\circ} \mathrm{C}$ for $15 \mathrm{sec}$ and $60^{\circ} \mathrm{C}$ for $1 \mathrm{~min}$. GAPDH expression was used for normalization. The quantification was performed using the $2^{-\Delta \Delta \mathrm{Cq}}$ method (29). The primer sequences were as follows: KNG1-primer-forward (F), 5'-TGT GGA TGC TGC TCT GA A GA A ATA-3'; KNG1-primer-reverse (R), 5'-GTCAGAGCCAACCGTCTT AGTG-3'; CASR-primer-F, 5'-CTCTACGATTGCTGTGGT GGGA-3'; CASR-primer-R, 5'-CTGCTGGAGGAGGCATAA CTGA-3'; GAPDH-primer-F, 5'-GGAAGCTTGTCATCA ATGGAAATC-3'; and GAPDH-primer-R, 5'-TGATGACCC TTTTGGCTCCC-3'. All PCRs were performed in triplicate.

Statistical analysis. Statistical analysis was carried out using R software (version 3.6.1 https://www.r-project.org/). A paired $\mathrm{t}$-test was employed to compare gene expression patterns in CCRCC and normal tissues from TCGA database, and for RT-qPCR experimental validation. The log-rank test was applied to compare the outcomes of Kaplan-Meier analysis between high and low-risk patients. Wilcoxon test was used to compare two independent non-parametric samples, while the Kruskal-Wallis test was used for multiple independent non-parametric samples. The Pearson correlation analysis was used to study the expression correlation between two genes. A two-tailed $\mathrm{P}<0.05$ was considered to indicate a statistically significant difference.

\section{Results}

DEG identification using CCRCC GEO datasets. A multistep analysis was conducted based on selected GEO datasets. Significant DEGs and their biological characteristics were identified using integrated bioinformatics analysis (Fig. 1). Since paired tumor and normal tissues from the same patient should ideally be compared to find genetic alterations (30), only GEO datasets with paired tumor and normal controls were selected. In brief, there were a total of six GEO datasets with GEPs of CCRCC, including 235 paired tumor and normal tissues.

Following processing and normalization of gene expression data (Fig. S1), DEGs were screened in each GEO dataset with a cut-off value of a corrected $\mathrm{P}<0.05$ and $\mid \log _{2} \mathrm{FCl}>1$. Overall, the GSE16441 dataset included 3,138 DEGs $(1,484$ up- and 1,654 downregulated), the GSE36895 dataset 1,609 (724 up- and 885 downregulated), the GSE40435 1,108 (498 up- and 610 downregulated), the GSE46699 1,056 (516 upand 540 downregulated), the GSE66270 4,474 (2,334 up- and 2,140 downregulated) and the GSE71963 1,844 (799 up- and 1,045 downregulated). Volcano plots of DEGs in each dataset are presented in Fig. 2A.

$D E G$ selection using the RRA method. After screening DEGs in each dataset, all 6 gene lists were sorted according to $\log _{2} \mathrm{FC}$. The RRA method was then used to compare the ranked gene lists. In brief, this method was based on the hypothesis of 


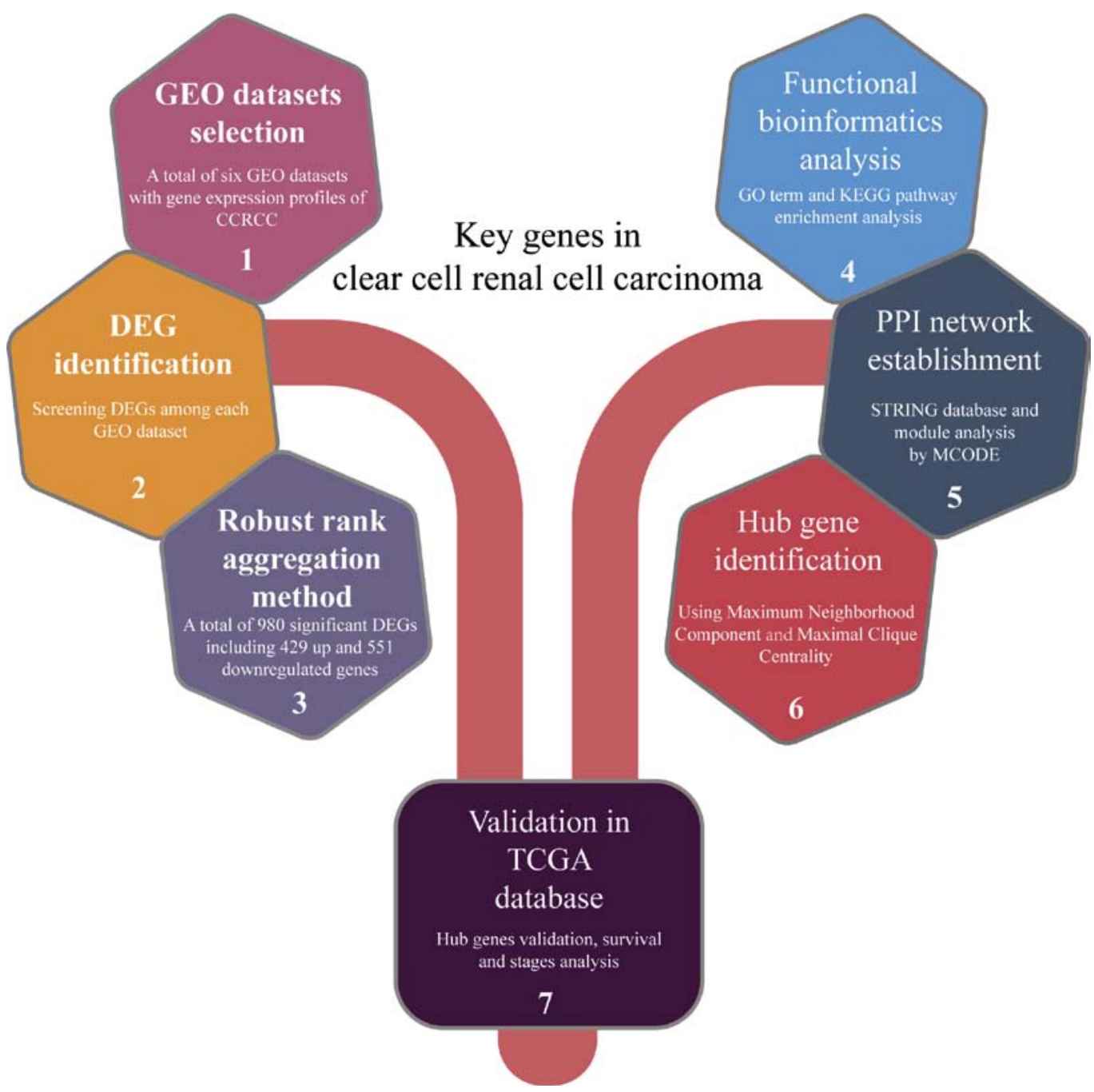

Figure 1. Workflow of the present study. Determination of potential key genes associated with CCRCC pathogenesis by integrated bioinformatics analysis. CCRCC, clear cell renal cell carcinoma; GEO, Gene Expression Omnibus; GO, Gene Ontology; DEGs, differentially expressed genes; TCGA, The Cancer Genome Atlas; PPI, protein-protein interaction; KEGG, Kyoto Encyclopedia of Genes and Genomes; STRING, Search Tool for the Retrieval of Interacting Genes/Proteins.

randomly arranged genes in each dataset. The higher the rank of a gene in all DEGs results, the smaller the P-value calculated by the RRA method, and the larger the possibility of robust DEGs. Based on this method, a total of 980 robust DEGs were obtained (429 up- and 551 downregulated; Table SII). A gene expression heatmap of the top 15 up- and downregulated DEGs is presented in Fig. 2B.

Functional enrichment analysis of DEGs. Next, the present study aimed to examine the biological functions associated with the aforementioned DEGs. DAVID (Table SIII) was used to conduct GO term enrichment analysis of integrated DEGs. The GO terms included BP, MF and CC. With regard to MF, upregulated DEGs were enriched in 'peptide antigen binding', 'MHC class II receptor activity' and 'receptor binding' (Table SIII). These genes were also clearly enriched in 'immune response', 'interferon-gamma-mediated signaling pathway' and BP 'inflammatory response' (Table SIII). With regard to CC, upregulated genes were enriched mainly in 'plasma membrane', 'extracellular space' and 'cell surface' (Fig. 3A). In addition, downregulated DEGs were enriched in 'oxidoreductase activity', 'anion:anion antiporter activity' and 'pyridoxal phosphate binding' in the MF group (Table SIII). With regard to $\mathrm{BP}$, these genes were enriched in 'oxidation-reduction process', 'gluconeogenesis' and 'metabolic process'. With regard to $\mathrm{CC}$, these genes were mainly enriched in 'extracellular exosome' and 'basolateral plasma membrane' (Fig. 3B).

Using KEGG pathway enrichment analysis based on KOBAS, upregulated genes exhibited an enrichment in 'apoptosis)', 'AMPK signalling pathway', as well as 'Jak-STAT signalling pathway' (Fig. 3C). In addition, these genes were closely associated with various signaling pathways, such as the HIF-1, PI3K-Akt, Rap1, NF- $\kappa$ B and p53 (Table SIII). The downregulated DEGs were mainly associated with metabolism, such as 'carbon metabolism', 'metabolic pathways', 'valine, leucine, isoleucine degradation', as well as 'glycine, serine and threonine metabolism' (Fig. 3D). According to these results, these DEGs exhibited a marked enrichment in cancer-related biological function.

PPI network construction and module analysis. The STRING database was used to construct a PPI network with a high 

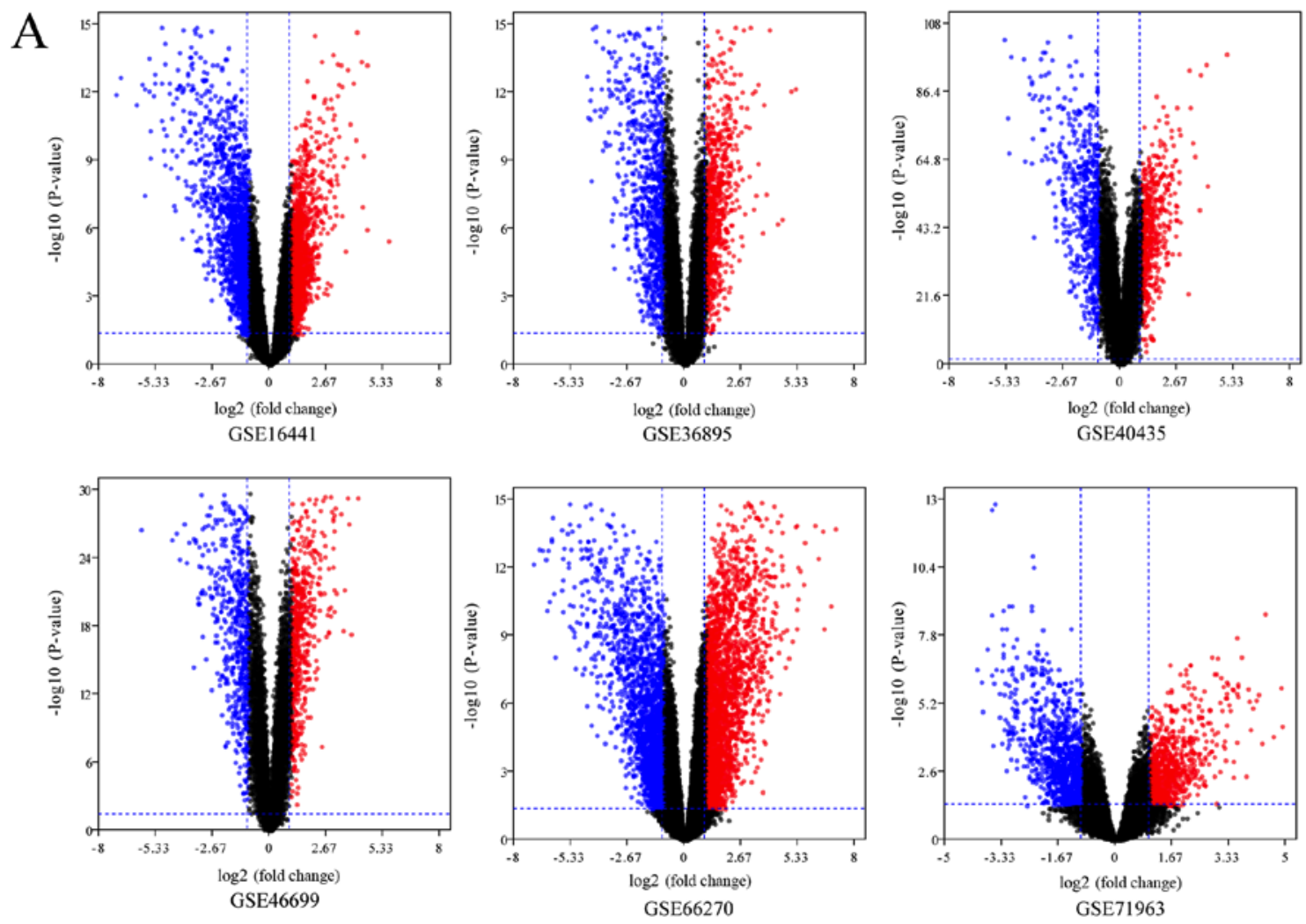

$\mathrm{B}$

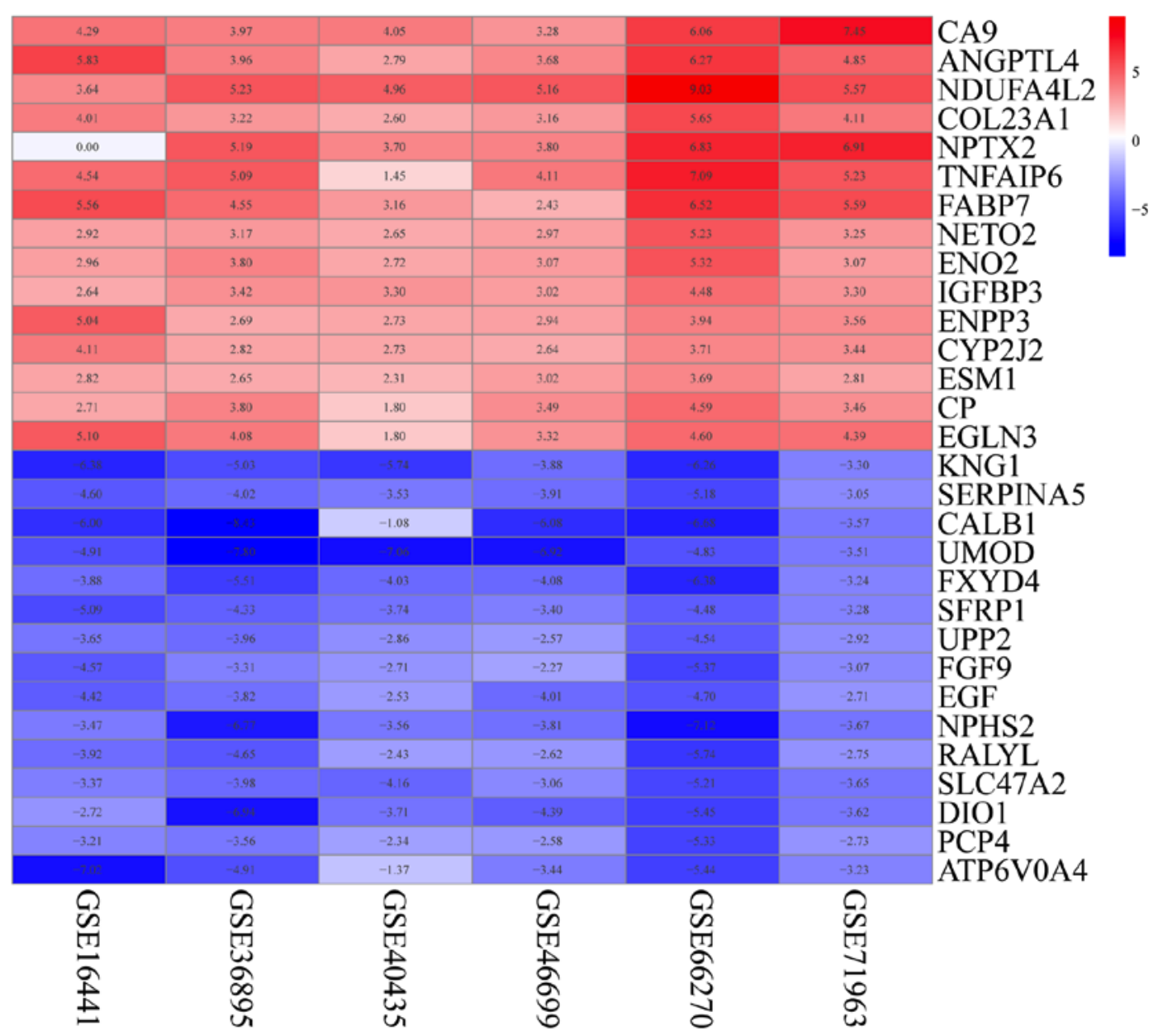

Figure 2. Determination of DEGs in every dataset using the RRA method in clear cell renal cell carcinoma. (A) Volcano plot of DEGs in each dataset. Red dots represent upregulated genes, blue dots represent downregulated genes and black dots represent genes with no significant difference in expression. (B) Expression heat map of the top 15 up- and downregulated DEGs, as determined using the RRA method. Gene Expression Omnibus datasets are arranged by column. Genes are arranged by row. Value in each box is the $\log _{2}$ (fold-change) value. DEGs, differentially expressed genes; RRA, robust rank aggregation. 
A

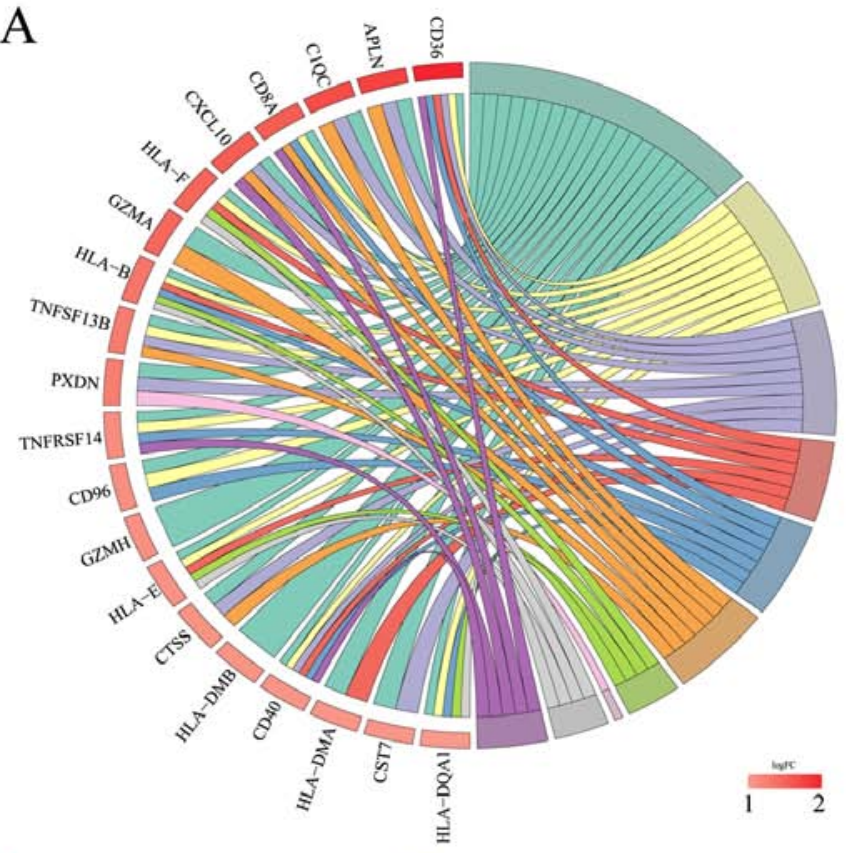

D plasma membrane E-extracellular region

Extracellular space
integral component of lumenal side of endoplasmic reticulum membrane

B
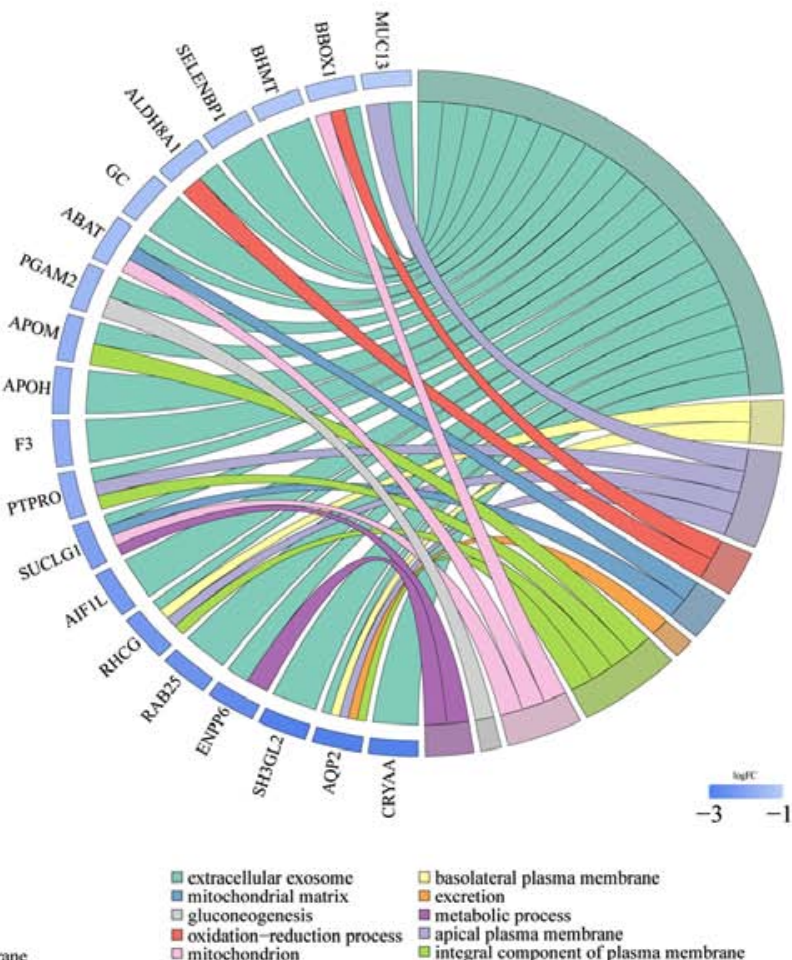

$\mathrm{C}$

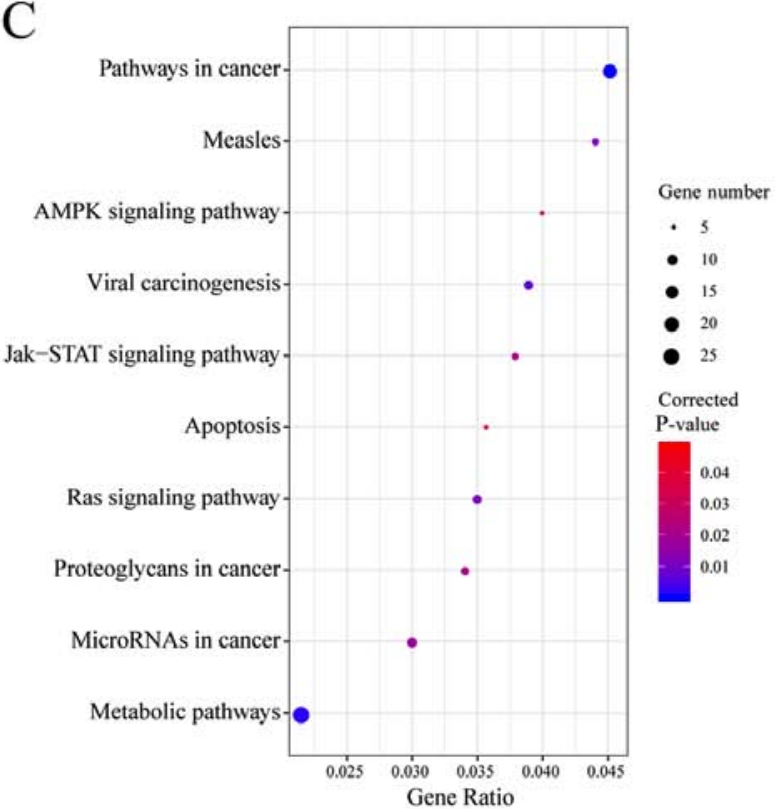

$\mathrm{D}$

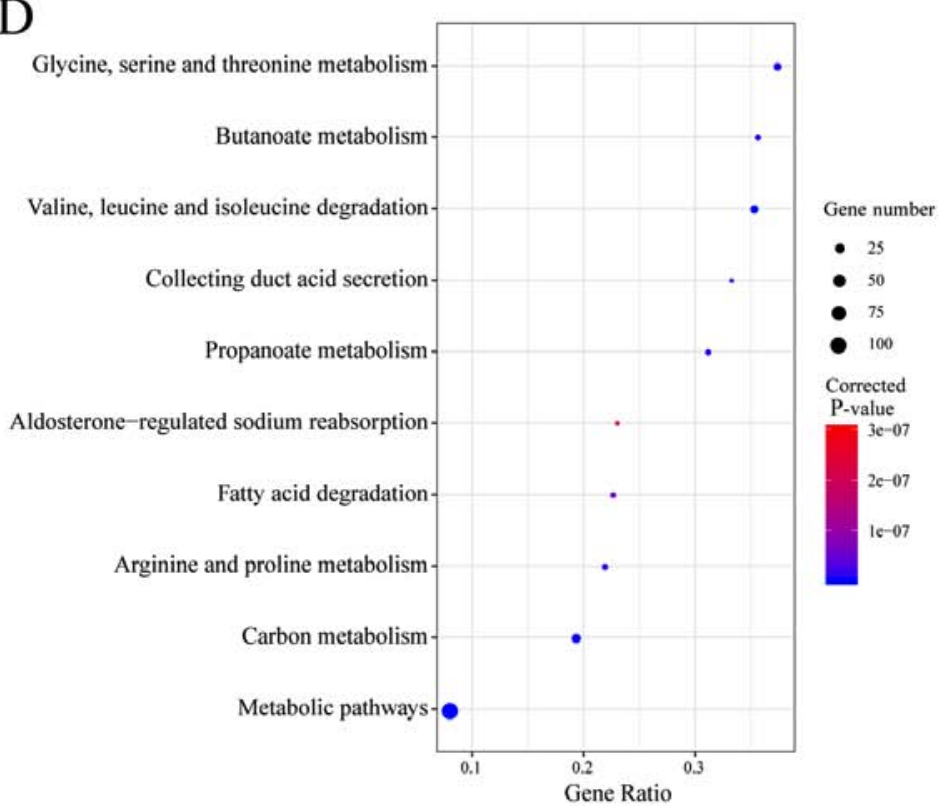

Figure 3. DEG functional enrichment analysis. Gene Ontology enrichment analysis of (A) upregulated and (B) downregulated DEGs. Kyoto Encyclopedia of Genes and Genomes pathway enrichment analysis of (C) upregulated and (D) downregulated DEGs. DEGs, differentially expressed genes.

confidence score $\geq 0.90$, which included 1,154 edges and 429 nodes (205 up- and 224 downregulated genes; Table SIV), as presented in (Fig. 4A; Table SV).

MCODE was used to identify four significant modules from the whole PPI network (Fig. 4B-E), with an enrichment of DEGs in module 1 in 'complement and coagulation cascades', 'chemokine signaling pathway' and 'cytokine-cytokine receptor interaction'. An apparent enrichment of genes in module 2 was observed in 'cell adhesion molecules (CAMs)', 'antigen processing and presentation' and 'phagosome'. An apparent enrichment of genes in module 3 was observed in 'protein digestion and absorption', 'ECM-receptor interaction' and 'focal adhesion'. Finally, an apparent enrichment of genes was observed in module 4 in 'ubiquitin mediated proteolysis', 'aldosterone-regulated sodium reabsorption' and 'protein processing in endoplasmic reticulum' (Table SVI).

Hub gene selection from the entire PPI network. Using the cytoHubba plug-in of Cytoscape software, hub genes were identified according to the MCC and MNC algorithms. A total 

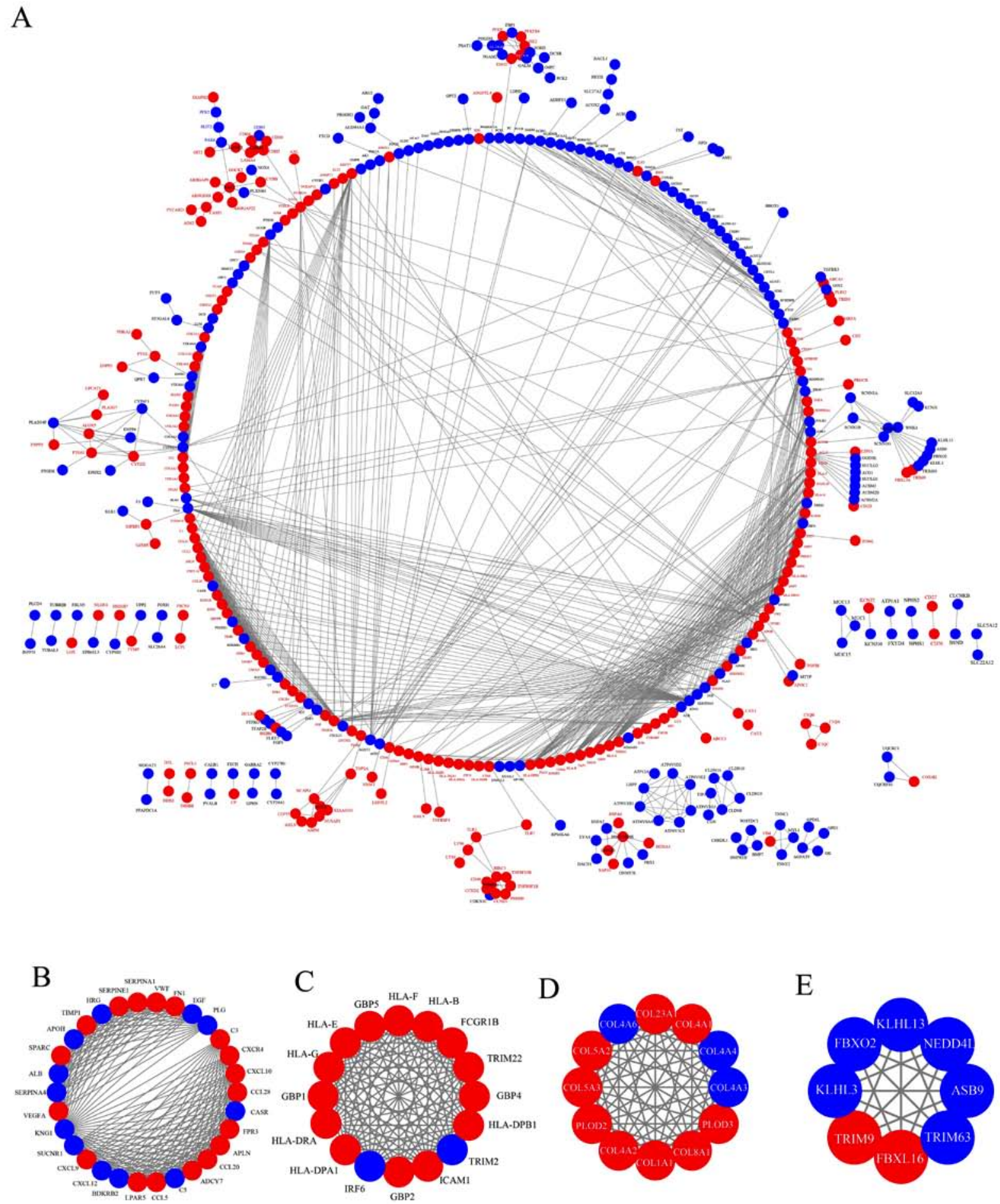

Upregulated genes

Downregulated genes

Figure 4. PPI network construction and module analysis. (A) Complete PPI network. PPI network modules (B) 1, (C) 2, (D) 3 and (E) 4. PPI, protein-protein interaction.

of six mutual hub genes were found using the two methods, as demonstrated by a Venn diagram: CXCL12, BDKRB2, ADCY7, CASR, KNG1 and LPAR5 (Fig. 5A; Table I). Therefore, these significant hub genes could be promising and potential biomarkers, as well as diagnostic and prognostic targets for CCRCC.
TCGA database validation and prognostic significance of hub genes. TCGA database was used to validate integrated DEG results based on the RNA sequencing data. First, the GEPs of the aforementioned six hub genes helped obtain 72 pairs of CCRCC and normal samples from TCGA database. These six hub genes were also found to be significantly differentially 
Table I. Hub genes identified from the Maximal Clique Centrality and Maximum Neighborhood Component methods using Venn diagram.

\begin{tabular}{lrlrl}
\hline Gene & Entrez & \multicolumn{1}{c}{ Full gene name } & $\log _{2} \mathrm{FC}$ & Expression \\
\hline ADCY7 & 113 & Adenylate cyclase 7 & 1.030452496 & Upregulated \\
LPAR5 & 57121 & Lysophosphatidic acid receptor 5 & 1.194101348 & Upregulated \\
CXCL12 & 6387 & C-X-C motif chemokine ligand 12 & -1.430728025 & Downregulated \\
BDKRB2 & 624 & Bradykinin receptor B2 & -1.115489479 & Downregulated \\
CASR & 846 & Calcium-sensing receptor & -1.704317596 & Downregulated \\
KNG1 & 3827 & Kininogen 1 & -5.140318003 & Downregulated \\
\hline
\end{tabular}

FC, fold-change.

expressed in the TCGA database $(\mathrm{P}<0.001)$, which was consistent with the GEO dataset results (Fig. 5B and C).

The GEPIA online analysis tool was used to examine the prognosis of the aforementioned hub genes using the GEPs from TCGA database. Only CASR exhibited a marked association with the overall survival (OS) of patients with CCRCC (log-rank P=0.027; Fig. 5D). Furthermore, BDKRB2 $(\mathrm{P}=0.0125)$, CASR $(\mathrm{P}=0.00433)$ and $\mathrm{KNG} 1(\mathrm{P}=0.0305)$ were differentially expressed at different stages of CCRCC (Fig. 5E).

To examine the association among these six hub genes, Pearson's correlation coefficient was used (Fig. S2). KNG1 positively correlated with CASR $(\mathrm{R}=0.92)$ and CXCL12 $(\mathrm{R}=0.51)$. However, KNG1 was negatively correlated with ADCY7 ( $\mathrm{R}=-0.53)$ and LPAR5 ( $\mathrm{R}=-0.55)$.

Moreover, in order to find clinical use of the hub genes, correlation analysis based on TCGA data and clinical information was performed (Fig. S3). Gene expression of LPAR5 $(\mathrm{P}=0.019)$, BDKRB2 $(\mathrm{P}<0.001)$ and CASR $(\mathrm{P}=0.016)$ was associated with sex. In addition, BDKRB2 $(\mathrm{P}=0.016)$ and CASR $(P=0.002)$ were associated with T stage. LPAR5 was associated with $\mathrm{N}$ stage $(\mathrm{P}=0.004)$ and $\mathrm{M}$ stage $(\mathrm{P}=0.009)$. ADCY7 $(\mathrm{P}=0.010)$ and CASR $(\mathrm{P}=0.006)$ were closely associated with $\mathrm{M}$ stage. Finally, LPAR5 $(\mathrm{P}=0.039)$ and CASR $(\mathrm{P}=0.009)$ were associated with histological grade.

Experimental validation of hub genes by RT-qPCR. In order to validate the results of identified hub genes, experimental validation was performed using RT-qPCR in 20 pairs of CCRCC and matched normal tissues $(n=40)$. The clinical information of patients with CCRCC in the validation experiment is presented in Table II. In the validation experiments, the two hub genes with the most significant changes, CASR and KNG1, were selected. The expressions of both genes were downregulated in tumor tissues, compared with matched normal tissues $(\mathrm{P}<0.01$; Fig. 6$)$, suggesting that the results obtained using bioinformatics analysis were consistent with experimental validation.

\section{Discussion}

In the present study, a comprehensive bioinformatics analysis was conducted for the identification of robust DEGs that could become potential biomarkers of CCRCC. According to
GEPs of GEO datasets, the RRA method was used to identify 980 markedly robust DEGs (429 upregulated genes and 551 downregulated). Functional biological analysis showed that these DEGs were clearly enriched in cancer-related biological functions and pathways. The STRING database was used for the construction of the PPI network. The Cytoscape MCODE plug-in was used to analyze modules from the entire network. In total, six hub genes were identified, including CXCL12, BDKRB2, ADCY7, CASR, KNG1 and LPAR5, which were verified in the TCGA database. Moreover, survival analysis suggested that CASR could affect the prognosis of patients with CCRCC.

Based on microarrays, RNA-sequencing technology and public databases, such as GEO, several gene expression studies on human cancer have been conducted in the last decade, including CCRCC. Chen et al (9) used two GEO microarray datasets of 101 tumor and 95 normal kidney samples to identify 2,425 DEGs (1,259 up- and 1,166 downregulated). In addition, the sva R package (31) was used to eliminate batch effects between datasets GSE36895 and GSE53757. The weighted gene co-expression network and module preservation analysis were carried out to identify 11 co-expressed gene modules. According to the functional enrichment analysis, the patho-module BPs were involved in biological pathways and processes associated with cell cycle and division. A total of 29 hub genes were finally identified, which exhibited a stronger association with CCRCC progression (9). In another previous CCRCC study, Song et al (7) used four GEO datasets containing 244 matched primary tumor and adjacent normal tissues. DEGs were identified in each dataset, and 424 aberrantly expressed mRNAs that were shared among these datasets were identified. Following integration with TCGA data, 252 shared genes with aberrant expression in both the GEO and TCGA datasets were identified. Similarly, 330 up- and 545 downregulated DEGs were found to overlap in 3 GEPs from GEO CCRCC datasets (32). A total of 8 DEGs were identified as biomarkers, including VEGFA, peroxisome proliferator activated receptor $\alpha$, cyclin D1, fms related receptor tyrosine kinase 1, CXCL12, fibronectin 1, decorin and erb-b2 receptor tyrosine kinase 4.

However, due to the heterogeneity of individuals, paired tumor and normal tissues from the same patient should ideally be compared to find genetic alterations (30). Thus, only GEO 
A

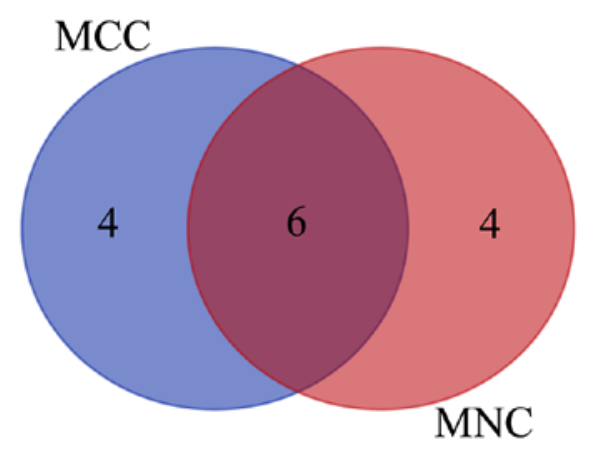

B

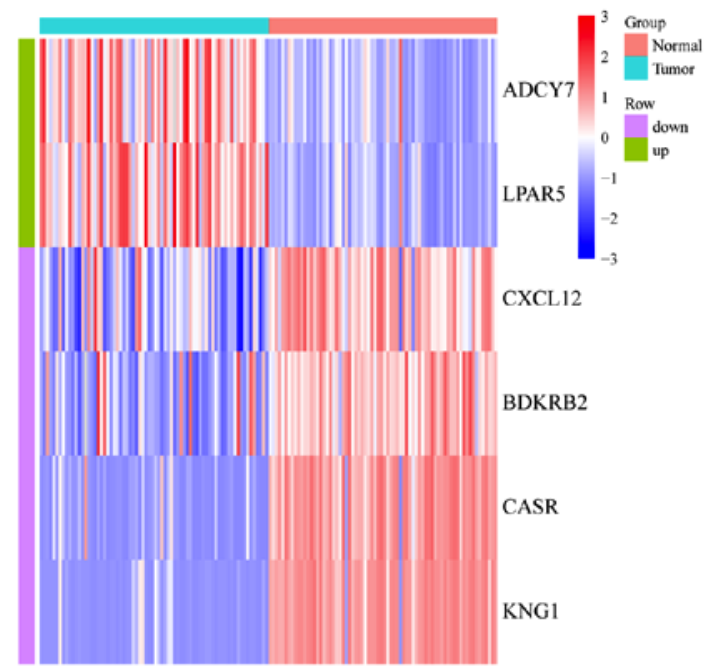

C

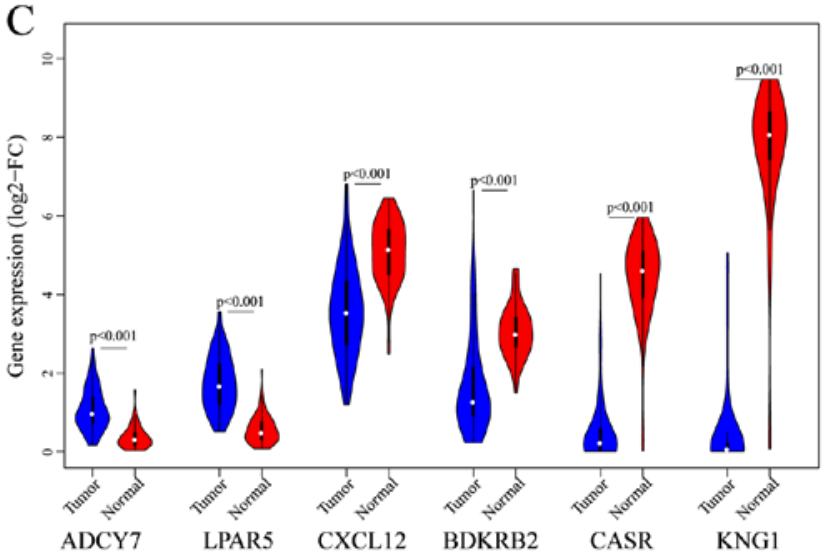

D

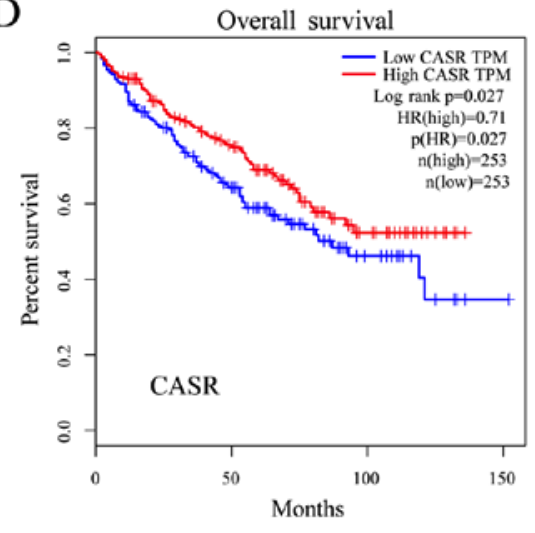

E

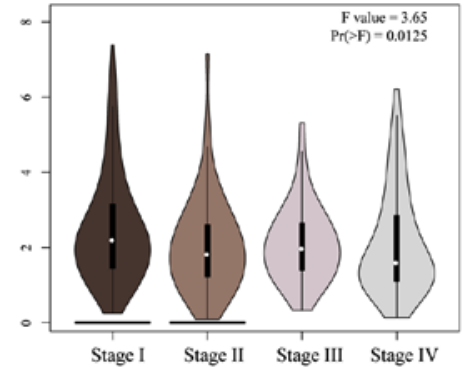

BDKRB2

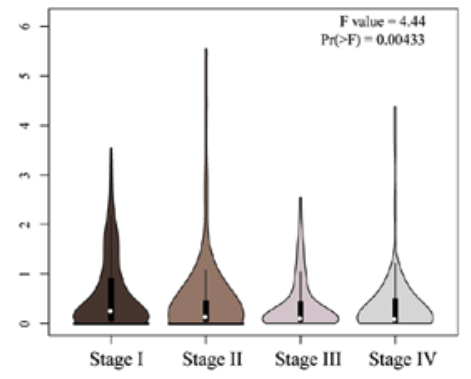

CASR

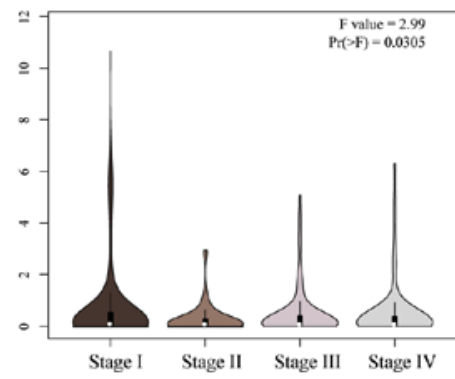

KNG1

Figure 5. TCGA database validation and prognostic significance of hub genes. (A) Venn diagram of shared hub genes, as determined using the MCC and MNC methods. (B) Gene expression heat map of six hub genes in TCGA database. (C) Violin plot of six hub genes in TCGA database. (D) Kaplan-Meier survival analysis according to CASR gene expression. (E) Gene expression of BDKRB2, CASR and KNG1 at different clear cell renal cell carcinoma stages. TCGA, The Cancer Genome Atlas; ADCY7, adenylate cyclase 7; LPAR5, lysophosphatidic acid receptor 5; CXCL12, C-X-C motif chemokine ligand 12; BDKRB2, bradykinin receptor B2; CASR, calcium-sensing receptor; KNG1, kininogen 1; HR, hazard ratio; MCC, Maximal Clique Centrality; MNC, Maximum Neighborhood Component; FC, fold-change; TPM, transcript per million.

datasets with paired tumor and normal tissues were selected in the present study. In addition, the methods used in the aforementioned studies were not suitable for various datasets, different platforms or limited sample sizes. By contrast, the RRA method used in the present study was suitable for the comparison of ranked gene lists, based on a hypothesis of randomly ordered genes. The higher the rank of a gene in all experiments, the smaller the P-value, and the larger the likelihood that the gene is a DEG. This method has been increasingly used recently in different types of human cancer. In one study of non-small cell lung cancer, Ni et al (13) analyzed four GEO datasets using the RRA method for gene integration, and identified 249 DEGs (113 up- and 136 downregulated); the 5 hub genes with the strongest connectivity were identified as CCNB1, TOP2A, ubiquitin conjugating enzyme E2 C (UBE2C), CCNA2 and kinesin family member 20A. Gao et al (12) identified 343 DEGs (111 upregulated and 232 downregulated) using the RRA method on five GEO datasets of bladder cancer to identify 10 
Table II. Clinical information of patients with clear cell renal cell carcinoma in the validation experiment.

\begin{tabular}{lccc}
\hline Clinicopathological parameter & Alive, $\mathrm{n}=16, \mathrm{n}(\%)$ & Dead, $\mathrm{n}=4, \mathrm{n}(\%)$ & Total, $\mathrm{n}=20, \mathrm{n}(\%)$ \\
\hline Sex & & & $10(50)$ \\
Female & $10(62.5)$ & $0(0)$ & $10(50)$ \\
Male & $6(37.5)$ & $4(100)$ & $57(11.1)$ \\
Age, mean (SD) & $56.8(9.6)$ & $58.2(17.8)$ & $56.5(42-79)$ \\
Age, median (range) & $56.5(44-76)$ & $56(42-79)$ & $12(60)$ \\
Stage & & & $3(15)$ \\
Stage I & $9(56.2)$ & $3(75)$ & $4(20)$ \\
Stage II & $3(18.8)$ & $0(0)$ & $1(5)$ \\
Stage III & $3(18.8)$ & $1(25)$ & $0(0)$ \\
Stage IV & $1(6.2)$ & & \\
\hline
\end{tabular}

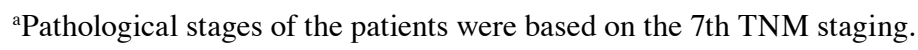
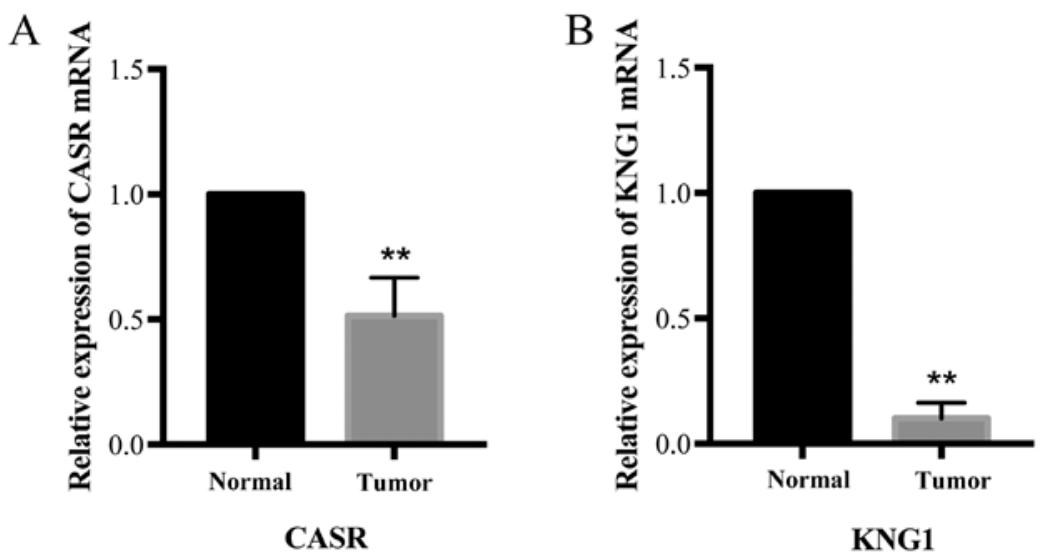

Figure 6. Experimental validation of hub genes by reverse transcription-quantitative PCR. (A) Gene expression of CASR in tumor and normal tissues. (B) Gene expression of KNG1 in tumor and normal tissues. All hub gene expressions were normalized against GAPDH expression. Statistical significance of differences between groups was calculated using Student's t-test. ${ }^{* *} \mathrm{P}<0.01$ vs. normal. CASR, calcium-sensing receptor; KNG1, kininogen 1.

hub genes: CCNB1, VEGFA, actin $\alpha 2$, TOP2A, aurora kinase (AURK) B, cell division cycle 20, AURKA, centrosomal protein 55 (CEP55), CCNB2 and UBE2C. In a gastric cancer study, Liu et al (11) merged nine GEO datasets and used the RRA method. A total of 9 hub genes that could affect the pathogenesis of gastric cancer were identified, namely TOP2A, cyclin-dependent kinase inhibitor 3 , collagen type I $\alpha 1$ chain (COL1A1), NDC80 kinetochore complex component, TPX2 microtubule nucleation factor, collagen type III $\alpha 1$ chain, CEP55, collagen type I $\alpha 2$ chain and tissue inhibitor of metallopeptidase 1 . Other previous studies, such as one focusing on ovarian cancer (14) and hepatocellular carcinoma (33) have also used this method.

The integrated bioinformatics analysis identified six important hub genes, CXCL12, ADCY7, BDKRB2, LPAR5, CASR and KNG1. CXCL12 is a member of the C-X-C motif chemokine subfamily. CXCL12/C-X-C motif chemokine receptor 4 signaling is known to affect tumor growth; therefore, targeting this pathway could serve as a new treatment strategy against cancer (34). CXCL12 exhibited a markedly higher expression in lung metastases, as compared with paired primary lesions of colorectal cancer (35). BDKRB2 has also been detected in numerous types of human cancer. Wei et al (36) screened potential biomarkers of the chemoresistant ovarian carcinoma, including six essential genes (phosphoinositide-3-kinase regulatory subunit 5, phosphatase and tensin homolog, BDKRB2, MAPK3, sphingosine-1-phosphate receptor 3 and nuclear cap binding protein subunit 2). In addition, microRNA (miRNA)-129-1-3p could target BDKRB2 in gastric cancer, thereby suppressing cell migration (37). The expression of ADCY7 was shown to be inversely correlated with the OS of patients with acute myeloid leukemia (38). CASR promoted bone metastasis in RCC (39) and was also identified as a hub gene in CCRCC by co-expression analysis (8). Overexpression of KNG1 promoted apoptosis and $\mathrm{G}_{1}$ phase cell cycle arrest, whilst suppressing the PI3K/Akt pathway in glioma cells (40). This gene was also reported in thyroid (41) and colorectal cancer (42), as well as tongue squamous cell carcinoma (43). LPAR5 was identified as a hub gene in thyroid cancer (41). LPAR5 was downregulated in primary nasopharyngeal carcinoma tissues, which, in turn, promoted lysophosphatidic acid-induced cell migration (44).

In the present study, 20 pairs of $\mathrm{CCRCC}$ and matched normal tissues were used for validation experiments. The 
results obtained from the experimental validation were consistent with the results obtained from the bioinformatics analysis. The sex distribution, age range as well as tumor stages observed in the patient recruited in the present study were similar to the clinical information obtained from public databases. Most of the datasets originated from Europe and America, and only one dataset was from Japan. However, patients with CCRCC with different geographical backgrounds also have different molecular biology backgrounds (45). Therefore, future studies will need to include datasets from other regional backgrounds, including for instance, the Chinese population.

In addition, future research of RCC should be focused on new methods or gene models. By literature searching, for example, a recent trend of developing computational models to identify some important RNAs (non-coding RNAs or miRNAs) related to disease was increasingly reported (46). New models have been established and implemented to identify novel miRNA-disease associations (47). For example, Chen et al (48) presented a novel model of Inductive Matrix Completion for miRNA-Disease Association prediction, with the aim of predicting unknown miRNA-disease associations based on known associations between integrated miRNA and disease similarity. Moreover, a matrix decomposition and heterogeneous graph inference calculation model for miRNA disease association prediction, which identifies new miRNA disease associations by integrating predictive association probabilities obtained from matrix decomposition through sparse learning methods, miRNA functional similarity, as well as other theories, was developed (49). Another previous study also developed a method of Laplacian Regularized Least Squares for lncRNA-Disease Association in the semi-supervised learning framework (50). The aforementioned studies may provide insight into the characterization of disease-related biomarkers.

To the best of the authors' knowledge, the present study is the first to use the RRA algorithm in the study of CCRCC. In addition, bioinformatics analysis was used to identify key genes in the development and progression of CCRCC. However, there are several limitations to the present study. Firstly, a larger sample size needs to be validated to obtain reliable results in further study. Moreover, novel and effective experimental methods and computational models need to be designed. Lastly, further research on hub genes needs to be carried out to examine molecular function or biological behavior in the occurrence and development of CCRCC.

In conclusion, GEO datasets were used in the present study to screen for robust DEGs that could be involved in CCRCC carcinogenesis. An integrated bioinformatics analysis was also performed. The present study identified reliable molecular biomarkers for the screening, diagnosis and prognosis of CCRCC, which may also serve as new therapeutic targets.

\section{Acknowledgements}

Not applicable.

\section{Funding}

The present study was supported by The National Science and Technology Major Project of the Ministry of Science and Technology of China (grant no. 2017ZX09304025), The Science and Technology Plan Project of Liaoning Province (grant no. 2016007010) and The General Projects of Liaoning Province Colleges and Universities (grant no. LFWK201706).

\section{Availability of data and materials}

All data generated or analyzed during the current study are included in this published article.

\section{Authors' contributions}

HC and LX conceived and designed the present study. HC and LX performed the analysis. ZL, KZH, XFC and BFL contributed to the literature search and did the initial analysis of the GEO data. HC and XJQ wrote the initial draft of the manuscript. XJQ and YPL assisted with the study design, obtained funding and also revised the manuscript critically for important intellectual content.. All authors read and approved the final version of the manuscript.

\section{Ethics approval and consent to participate}

Informed consent forms were signed by all patients prior to sample collection. The present study was approved by The Research Ethics Committee of China Medical University (approval no. 2015PS44K).

\section{Patient consent for publication}

Not applicable.

\section{Competing interests}

The authors declare that they have no competing interests.

\section{References}

1. Hsieh JJ, Purdue MP, Signoretti S, Swanton C, Albiges L, Schmidinger M, Heng DY, Larkin J and Ficarra V: Renal cell carcinoma. Nat Rev Dis Primers 3: 17009, 2017.

2. Siegel RL, Miller KD and Jemal A: Cancer Statistics, 2017. CA Cancer J Clin 67: 7-30, 2017.

3. Ljungberg B, Bensalah K, Canfield S, Dabestani S, Hofmann F, Hora M, Kuczyk MA, Lam T, Marconi L, Merseburger AS, et al: EAU guidelines on renal cell carcinoma: 2014 update. Eur Urol 67: 913-924, 2015.

4. Tosco L, Van Poppel H, Frea B, Gregoraci G and Joniau S: Survival and impact of clinical prognostic factors in surgically treated metastatic renal cell carcinoma. Eur Urol 63: 646-652, 2013.

5. Grignon DJ and Che M: Clear cell renal cell carcinoma. Clin Lab Med 25: 305-316, 2005.

6. Kim HS, Kim JH, Jang HJ, Han B and Zang DY: Clinicopathologic significance of VHL gene alteration in clear-cell renal cell carcinoma: An updated meta-analysis and review. Int J Mol Sci 19: pii: E2529, 2018

7. Song E, Song W, Ren M, Xing L, Ni W, Li Y, Gong M, Zhao M, Ma X, Zhang X and An R: Identification of potential crucial genes associated with carcinogenesis of clear cell renal cell carcinoma. J Cell Biochem 119: 5163-5174, 2018.

8. Yuan L, Zeng G, Chen L, Wang G, Wang X, Cao X, Lu M, Liu X, Qian G, Xiao Y and Wang X: Identification of key genes and pathways in human clear cell renal cell carcinoma (ccRCC) by co-expression analysis. Int J Biol Sci 14: 266-279, 2018.

9. Chen L, Yuan L, Qian K, Qian G, Zhu Y, Wu CL, Dan HC, Xiao $\mathrm{Y}$ and Wang $\mathrm{X}$ : Identification of biomarkers associated with pathological stage and prognosis of clear cell renal cell carcinoma by co-expression network analysis. Front Physiol 9: 399, 2018 
10. Kolde R, Laur S, Adler P and Vilo J: Robust rank aggregation for gene list integration and meta-analysis. Bioinformatics 28 : 573-580, 2012.

11. Liu X, Wu J, Zhang D, Bing Z, Tian J, Ni M, Zhang X, Meng Z and Liu S: Identification of potential key genes associated with the pathogenesis and prognosis of gastric cancer based on integrated bioinformatics analysis. Front Genet 9: 265, 2018.

12. Gao X, Chen Y, Chen M, Wang S, Wen X and Zhang S: Identification of key candidate genes and biological pathways in bladder cancer. PeerJ 6: e6036, 2018.

13. Ni M, Liu X, Wu J, Zhang D, Tian J, Wang T, Liu S, Meng Z, Wang K, Duan X, et al: Identification of candidate biomarkers correlated with the pathogenesis and prognosis of non-small cell lung cancer via integrated bioinformatics analysis. Front Genet 9: 469, 2018.

14. Yang X, Zhu S, Li L, Zhang L, Xian S, Wang Y and Cheng Y: Identification of differentially expressed genes and signaling pathways in ovarian cancer by integrated bioinformatics analysis. Onco Targets Ther 11: 1457-1474, 2018.

15. Liu H, Brannon AR, Reddy AR, Alexe G, Seiler MW, Arreola A, Oza JH, Yao M, Juan D, Liou LS, et al: Identifying mRNA targets of microRNA dysregulated in cancer: With application to clear cell renal cell carcinoma. BMC Syst Biol 4: 51, 2010.

16. Peña-Llopis S, Vega-Rubin-de-Celis S, Liao A, Leng N, Pavía-Jiménez A, Wang S, Yamasaki T, Zhrebker L, Sivanand S, Spence P, et al: BAP1 loss defines a new class of renal cell carcinoma. Nat Genet 44: 751-759, 2012.

17. Wozniak MB, Le Calvez-Kelm F, Abedi-Ardekani B, Byrnes G, Durand G, Carreira C, Michelon J, Janout V, Holcatova I, Foretova $\mathrm{L}$, et al: Integrative genome-wide gene expression profiling of clear cell renal cell carcinoma in Czech Republic and in the United States. PLoS One 8: e57886, 2013.

18. Eckel-Passow JE, Serie DJ, Bot BM, Joseph RW, Cheville JC and Parker AS: ANKS1B is a smoking-related molecular alteration in clear cell renal cell carcinoma. BMC Urol 14: 14, 2014.

19. Wotschofsky Z, Gummlich L, Liep J, Stephan C, Kilic E, Jung K, Billaud JN and Meyer HA: Integrated microRNA and mRNA signature associated with the transition from the locally confined to the metastasized clear cell renal cell carcinoma exemplified by miR-146-5p. PLoS One 11: e0148746, 2016.

20. Takahashi M, Tsukamoto Y, Kai T, Tokunaga A, Nakada C, Hijiya N,Uchida T, Daa T, Nomura T, Sato F, et al: Downregulation of WDR20 due to loss of $14 \mathrm{q}$ is involved in the malignant transformation of clear cell renal cell carcinoma. Cancer Sci 107: 417-423, 2016

21. Ritchie ME, Phipson B, Wu D, Hu Y, Law CW, Shi W and Smyth GK: limma powers differential expression analyses for RNA-sequencing and microarray studies. Nucleic Acids Res 43: e47, 2015.

22. Huang da W, Sherman BT and Lempicki RA: Systematic and integrative analysis of large gene lists using DAVID bioinformatics resources. Nat Protoc 4: 44-57, 2009.

23. Xie C, Mao X, Huang J, Ding Y, Wu J, Dong S, Kong L, Gao G, Li CY and Wei L: KOBAS 2.0: A web server for annotation and identification of enriched pathways and diseases. Nucleic Acids Res 39 (Web Server Issue): W316-W322, 2011.

24. Szklarczyk D, Morris JH, Cook H, Kuhn M, Wyder S, Simonovic M, Santos A, Doncheva NT, Roth A, Bork P, et al: The STRING database in 2017: Quality-controlled protein-protein association networks, made broadly accessible. Nucleic Acids Res 45 (D1): D362-D368, 2017.

25. Bader GD and Hogue CW: An automated method for finding molecular complexes in large protein interaction networks. BMC Bioinformatics 4: 2, 2003.

26. Chin CH, Chen SH, Wu HH, Ho CW, Ko MT and Lin CY: cytoHubba: Identifying hub objects and sub-networks from complex interactome. BMC Syst Biol 8 (Suppl 4): S11, 2014.

27. Tang Z, Li C, Kang B, Gao G, Li C and Zhang Z: GEPIA: A web server for cancer and normal gene expression profiling and interactive analyses. Nucleic Acids Res 45 (W1): W98-W102, 2017.

28. Greene FL, Gospodarowicz MK and Wittekend C (eds): American Joint Committee On Cancer (AJCC) cancer staging manual. (7th edition). Philadelphia, Springer, 2009.

29. Livak KJ and Schmittgen TD: Analysis of relative gene expression data using real-time quantitative PCR and the 2(-Delta Delta C(T)) method. Methods 25: 402-408, 2001.
30. Ge S, Xia X, Ding C, Zhen B, Zhou Q, Feng J, Yuan J, Chen R, $\mathrm{Li} \mathrm{Y,} \mathrm{Ge} \mathrm{Z,} \mathrm{et} \mathrm{al:} \mathrm{A} \mathrm{proteomic} \mathrm{landscape} \mathrm{of} \mathrm{diffuse-type} \mathrm{gastric}$ cancer. Nat Commun 9: 1012, 2018.

31. Leek JT and Storey JD: Capturing heterogeneity in gene expression studies by surrogate variable analysis. PLoS Genet 3: $1724-1735,2007$

32. Wu F, Wu S and Gou X: Identification of biomarkers and potential molecular mechanisms of clear cell renal cell carcinoma. Neoplasma 65: 242-252, 2018.

33. Yang Y, Lu Q, Shao X, Mo B, Nie X, Liu W, Chen X, Tang Y, Deng Y and Yan J: Development of A three-gene prognostic signature for hepatitis B virus associated hepatocellular carcinoma based on integrated transcriptomic analysis. J Cancer 11: 989-2002, 2018.

34. Zhou Y,CaoHB,Li WJ and ZhaoL: The CXCL12 (SDF-1)/CXCR4 chemokine axis: Oncogenic properties, molecular targeting, and synthetic and natural product CXCR4 inhibitors for cancer therapy. Chin J Nat Med 16: 801-810, 2018.

35. Wang M, Yang X, Wei M and Wang Z: The role of CXCL12 axis in lung metastasis of colorectal cancer. J Cancer 9: 3898-3903, 2018.

36. Wei S, Wang Y, Xu H and Kuang Y: Screening of potential biomarkers for chemoresistant ovarian carcinoma with miRNA expression profiling data by bioinformatics approach. Oncol Lett 10: 2427-2431, 2015.

37. Wang D, Luo L and Guo J: miR-129-1-3p inhibits cell migration by targeting BDKRB2 in gastric cancer. Med Oncol 31: 98, 2014.

38. Li C, Xie J, Lu Z, Chen C, Yin Y, Zhan R, Fang Y, Hu X and Zhang CC: ADCY7 supports development of acute myeloid leukemia. Biochem Biophys Res Commun 465: 47-52, 2015.

39. Frees S, Breuksch I, Haber T, Bauer HK, Chavez-Munoz C, Raven P, Moskalev I, D Costa N, Tan Z, Daugaard M, et al: Calcium-sensing receptor (CaSR) promotes development of bone metastasis in renal cell carcinoma. Oncotarget 9: 15766-15779, 2018.

40. Xu J, Fang J, Cheng Z, Fan L, Hu W, Zhou F and Shen H: Overexpression of the Kininogen-1 inhibits proliferation and induces apoptosis of glioma cells. J Exp Clin Cancer Res 37: 180, 2018.

41. Tang J, Kong D, Cui Q, Wang K, Zhang D, Yuan Q, Liao X, Gong Y and Wu G: Bioinformatic analysis and identification of potential prognostic microRNAs and mRNAs in thyroid cancer. PeerJ 6: e4674, 2018.

42. Yu J, Huang Y, Lin C, Li X, Fang X, Zhong C, Yuan Y and Zheng S: Identification of kininogen 1 as a serum protein marker of colorectal adenoma in patients with a family history of colorectal cancer. J Cancer 9: 540-547, 2018.

43. Zhang H, Liu J, Fu X and Yang A: Identification of key genes and pathways in tongue squamous cell carcinoma using bioinformatics analysis. Med Sci Monit 23: 5924-5932, 2017.

44. Yap LF, Velapasamy S, Lee HM, Thavaraj S, Rajadurai P, Wei W, Vrzalikova K, Ibrahim MH, Khoo AS, Tsao SW, et al: Down-regulation of LPA receptor 5 contributes to aberrant LPA signalling in EBV-associated nasopharyngeal carcinoma. J Pathol 235: 456-465, 2015.

45. Znaor A, Lortet-Tieulent J, Laversanne M, Jemal A and Bray F: International variations and trends in renal cell carcinoma incidence and mortality. Eur Urol 67: 519-530, 2015.

46. Lopez R, Wang R and Seelig G: A molecular multi-gene classifier for disease diagnostics. Nat Chem 10: 746-754, 2018.

47. Chen X, Xie D, Zhao Q and You ZH: MicroRNAs and complex diseases: From experimental results to computational models. Brief Bioinform 20: 515-539, 2019

48. Chen X, Wang L, Qu J, Guan NN and Li JQ: Predicting miRNA-disease association based on inductive matrix completion. Bioinformatics 34: 4256-4265, 2018

49. Chen X, Yin J, Qu J and Huang L: MDHGI: Matrix decomposition and heterogeneous Graph inference for miRNA-disease association prediction. PLoS Comput Biol 14: e1006418, 2018.

50. Chen $X$ and Yan GY: Novel human lncRNA-disease association inference based on lncRNA expression profiles. Bioinformatics 29: 2617-2624, 2013.

This work is licensed under a Creative Commons Attribution-NonCommercial-NoDerivatives 4.0 International (CC BY-NC-ND 4.0) License. 